From the Division of Public Health Sciences, Fred Hutchinson Cancer Research Center, Seattle, WA; Section of General Internal Medicine, Washington Hospital Center, Washington, DC; Division of Research, Kaiser Permanente, Oakland; Stanford Prevention Research Center, Stanford University School of Medicine, Stanford; and Department of Medicine, Los Angeles Biomedical Research Institute at Harbor, UCLA Medical Center, Torrance, CA; Department of Preventive Medicine, State University of Stony Brook, Stony Brook, NY; Department of Public Health and Preventive Medicine, Oregon Health and Science University, Portland, OR; and Department of Obstetrics and Gynecology, University of Miami, Miller School of Medicine, Miami, FL.

Submitted July 8, 2009; accepted December 4, 2009; published online ahead of print at www.jco.org on January 25, 2010.

Supported in part by the National Heart, Lung, and Blood Institute, National Institutes of Health, US Department of Health and Human Services Contracts No. N01WH22110, 24152, 32100-2, 32105-6, 32108-9, 32111-13, 32115 $32118-32119,32122,42107-26$, 42129-32, and 44221 .

Authors' disclosures of potential conflicts of interest and author contributions are found at the end of this article.

Corresponding author: Christopher I. Li, $\mathrm{MD}, \mathrm{PhD}$, Fred Hutchinson Cancer

Research Center, $1100 \mathrm{~N}$ Fairview Ave, M4-C308, Seattle, WA 98109-1024:

e-mail: cili@fhcrc.org

(C) 2010 by American Society of Clinical Oncology

0732-183X/10/2806-1005/\$20.00

DOI: $10.1200 / J C O .2009 .25 .0423$

\title{
Migraine History and Breast Cancer Risk Among Postmenopausal Women
}

Christopher I. Li, Robert W. Mathes, Elizabeth C. Bluhm, Bette Caan, Mary F. Cavanagh, Rowan T. Chlebowski, Yvonne Michael, Mary Jo O'Sullivan, Marcia L. Stefanick, and Ross Prentice

\section{$\begin{array}{llllllll}\text { A } & \text { B } & \text { S } & \text { T } & \text { R } & \text { A } & \text { C } & \text { T }\end{array}$}

\section{Purpose}

Both migraine and breast cancer are hormonally mediated. Two recent reports indicate that women with a migraine history may have a lower risk of postmenopausal breast cancer than those who never suffered migraines. This finding requires confirmation; in particular, an assessment of the influence of use of nonsteroidal anti-inflammatory drugs (NSAID) is needed, because many studies indicate that NSAID use also may confer a reduction in breast cancer risk.

\section{Methods}

We assessed the relationship between self-reported history of migraine and incidence of postmenopausal breast cancer in 91,116 women enrolled on the Women's Health Initiative Observational Study prospective cohort from 1993 to 1998 at ages 50 to 79 years. Through September 15, 2005, there were 4,006 eligible patients with breast cancer diagnosed.

\section{Results}

Women with a history of migraine had a lower risk of breast cancer (hazard ratio [HR], 0.89; 95\% $\mathrm{Cl}, 0.80$ to 98 ) than women without a migraine history. This risk did not vary by recent NSAID use. The lower risk was somewhat more pronounced for invasive estrogen-receptor-positive and progesterone-receptor-positive tumors (HR, 0.83; 95\% Cl, 0.71 to 0.97), as no reduction in risk was observed for invasive ER-negative/PR-negative tumors (HR, 1.16; 95\% Cl, 0.86 to 1.57), and this difference in risk estimates was borderline statistically significant $(P=.06)$

\section{Conclusion}

This study supports the hypothesis that a history of migraine is associated with a lower risk of breast cancer and that this relationship is independent of recent NSAID use.

\section{J Clin Oncol 28:1005-1010. (C) 2010 by American Society of Clinical Oncology}

\section{INTRODUCTION}

Two recently published studies are the first reports that postmenopausal women with a clinical history of migraine have a $26 \%$ to $33 \%$ lower risk of invasive breast cancer than women without such a history. ${ }^{1,2}$ A potential link between migraine history and breast cancer risk was hypothesized, because both diseases are influenced by reproductive hormones. In women, the risk of migraine changes during menarche, menses, pregnancy, and perimenopause as a result of fluctuating estrogen levels. ${ }^{3}$ Particularly relevant to breast cancer risk is that migraines in women are often associated with declines in estrogen levels. Migraine frequency increases during menses, when endogenous estrogen levels drop sharply, and when the levels reach their lowest point in cycling premenopausal women. Specifically, approximately $60 \%$ of female migraineurs report a higher frequency and severity of migraines around the time of menses. ${ }^{4,5}$ Similarly, migraine frequency also increases during the hormone-free week of oral contraceptive use when estrogen is withdrawn. ${ }^{6}$ These observations suggest that marked drops in estrogen may be a migraine trigger. ${ }^{7}$ Indeed, $4 \%$ to $14 \%$ of women with migraine report that their migraines exclusively occur 2 days before to 3 days after the onset of menses. ${ }^{8-10}$ In contrast, two observations indicate that stable estrogen levels, whether they are high or low, are associated with a reduction in migraine frequency. First, during pregnancy, when women reach a high estrogen steady-state and do not experience monthly hormonal fluctuations, migraine frequency is reduced for almost all migraine sufferers. Approximately 11\% of migraine sufferers who become pregnant experience no migraines during pregnancy, and $87 \%$ experience a reduced frequency of migraine. ${ }^{11}$ Second, after women go through menopause and estrogen levels hold at a generally low steady-state, $67 \%$ of women 
with a history of premenopausal migraine report fewer migraines. ${ }^{12}$ Given that lifetime estrogen exposure is strongly related to breast cancer risk ${ }^{13}$ and that hormones clearly influence migraine, a relationship between these two health problems is biologically plausible. Additionally supporting this link is the observation that migraine history was more strongly related to a lower risk of hormone-receptor-positive breast cancer than it was to risk of hormone-receptor-negative disease in the two studies that have assessed this association. ${ }^{1,2}$

The paucity of information regarding the association between migraine and breast cancer warrants additional assessment of this relationship. One of the limitations of the two prior studies of migraine and breast cancer was the lack of data on use of nonsteroidal anti-inflammatory drugs (NSAIDs). This is relevant, because migraine sufferers are more likely to use these medications, and they are the only type of medication commonly used to treat migraines that has been shown to be modestly related to breast cancer risk. In particular, a recent meta-analysis of studies of NSAID use and breast cancer risk observed a $12 \%$ lower risk for women classified as ever using any NSAID. ${ }^{14}$ Similarly, in the Women's Health Initiative (WHI) Observational Study (OS) prospective cohort, regular use of any NSAID for 5 years or longer was associated with a 19\% lower risk of breast cancer compared with non-NSAID users and users of NSAIDs for less than 1 year. ${ }^{15}$ The WHI OS provides an excellent setting to evaluate the relationship between migraine and breast cancer risk because data on NSAID use and migraine history were collected, and because in this large cohort more than 4,000 patients with breast cancer have been diagnosed during cohort follow-up.

\section{METHODS}

This study utilized data collected in the WHI OS. The details of the scientific rationale, eligibility criteria, and design of the WHI OS have been published. ${ }^{16}$ Briefly, 93,676 postmenopausal women, age 50 to 79 years, were enrolled between October 1, 1993 and December 31, 1998, through 40 clinical centers dispersed throughout the United States, and these women were not subjected to any study interventions. All exposures used in this analysis were collected at the time of entry into the WHI OS. Data were collected from participants uniformly according to standardized institutional review board-approved procedures and protocols by centrally trained study staff. All participants provided written informed consent for participation in the WHI OS at the time of enrollment.

Cohort members completed baseline self-administered questionnaires covering a wide range of topics, including demographic characteristics, medical history, reproductive history, lifestyle characteristics, and family history of various diseases. In addition, baseline height and weight were measured by study staff. With respect to our primary exposure of interest, clinical history of migraine, women were asked at baseline if they have ever been told by a doctor that they had migraine headaches. The 2,560 women with an unknown migraine history were excluded from all analyses, so a total of 91,116 included women remained. Information about migraine was not assessed in subsequent questionnaires, so all analyses were based on migraine history at baseline. Participants also were asked at baseline to provide information on all over-thecounter and prescription drugs they were currently using and the durations they had been using them. These data were queried to identify women who were using NSAIDs at baseline, including aspirin, ibuprofen, naproxen, and various less commonly used over-the-counter and prescription-only NSAIDs. Summary variables of use of any NSAID at baseline and duration of current NSAID use were computed by using this information. Only women reporting NSAID use at least two times in each of the 2 weeks preceding completion of the baseline questionnaire were recorded as current NSAID users.
The primary follow-up of WHI OS participants was through annual mailed, self-administered questionnaires. This report includes information collected on the cohort through September 15, 2005, through which $2.2 \%$ of participants had been lost to follow-up, $2.5 \%$ declined additional follow-up, and $6.7 \%$ had died. Women with breast cancer were identified from annual questionnaires. The medical records of all women reporting a breast cancer diagnosis were reviewed by a study adjudicator at the respective study centers to verify the diagnosis. For the 4,006 confirmed patients identified through September 15, 2005 in the entire cohort, information from medical records was forwarded to the WHI coordinating center for coding of breast cancer stage, estrogen receptor (ER) status, progesterone receptor (PR) status, and histology. Of the 4,006 occurrences of breast cancer, 688 were in situ, and 3,318 were invasive. Analyses were also conducted that focused on subgroups of invasive breast cancer defined by ER/PR status and histology. In the ER/PRspecific analyses, the 609 invasive occurrences with unknown ER/PR status were excluded, as were those with ER-negative/PR-positive tumors, because of insufficient statistical power $(n=40)$, so that a total of 2,669 invasive cases remained for inclusion in the analysis. Analyses by invasive breast cancer histology were based on occurrences classified as ductal ( $\mathrm{n}=1,916$; International Classification of Disease, Oncology [ICD-O] -8500) or lobular ( $\mathrm{n}=754$; ICD-O-8520 and -8522). Those with other ICD-O codes were grouped together as having other histology $(\mathrm{n}=392)$, and 256 invasive occurrences had unknown histology.

\section{Statistical Analysis}

Cox regression was used to calculate hazard ratios (HRs) and 95\% CIs as measures of the association between migraine history and breast cancer risk. Time to breast cancer was computed from date of enrollment to date of first breast cancer diagnosis, and times for women without breast cancer were censored by date of last study follow-up or by September 15, 2005, whichever occurred first. All analyses were adjusted for age in years at enrollment (as a linear continuous term) and ethnicity (as a categoric term) through stratification of the baseline hazard rates in the Cox model, and women with no history of clinically diagnosed migraine served as the reference category. To additionally control for age at enrollment, we also adjusted all models for age at enrollment as a categoric variable (in 5-year categories). Variables considered potential confounders or effect modifiers included the following baseline characteristics: income, parity, use of menopausal hormone therapy, hysterectomy status, recent timing and duration of NSAID use, current use of migraine specific prescription medications, body mass index (BMI), smoking status, alcohol intake, and average number of cups of regular coffee consumed. Although several of these potential confounders have been associated with breast cancer risk, none of them changed our risk estimates by more than $10 \%$. However, we do present risk estimates from models adjusted simply for age and ethnicity and risk estimates from models additionally adjusted for the following baseline characteristics according to the way they are categorized in Table 1: hysterectomy history, use of menopausal hormones, NSAID use/ duration, alcohol consumption, smoking status, and consumption of caffeinated coffee. Effect modification was assessed by using likelihood ratio testing, and none of these variables were observed to be statistically significant effect modifiers (all $P$ for interaction $>.05$ ). Given the particular interest in the role of NSAID use, results stratified by NSAID use at enrollment are presented. All analyses were conducted by using Stata 9.2 (Stata Corp, College Station, TX).

\section{RESULTS}

Compared with women without a history of migraine, women with a clinical history of migraine as a group were slightly younger, more frequently non-Hispanic white, more likely to have used oral contraceptives and unopposed estrogen postmenopausal hormone therapy at enrollment, more likely to have had a hysterectomy, more commonly regular users of NSAIDs for 3 years or longer at the time of enrollment, and less likely to consume seven or more alcoholic beverages per week at baseline (Table 1). The two groups of women were 
Table 1. Distribution of Demographic and Lifestyle Characteristics Among Women With and Without a Clinical History of Migraine

\begin{tabular}{|c|c|c|c|c|}
\hline \multirow[b]{3}{*}{ Characteristic } & \multicolumn{4}{|c|}{ History of Migraine } \\
\hline & \multicolumn{2}{|c|}{$\begin{array}{c}\text { No } \\
(n=80,652)\end{array}$} & \multicolumn{2}{|c|}{$\begin{array}{c}\text { Yes } \\
(\mathrm{n}=10,464) \\
\end{array}$} \\
\hline & No. & $\%$ & No. & $\%$ \\
\hline \multicolumn{5}{|l|}{ Age at enrollment, years } \\
\hline $50-59$ & 24,608 & 30.5 & 4,225 & 40.4 \\
\hline $60-69$ & 35,709 & 44.3 & 4,373 & 41.8 \\
\hline $70-79$ & 20,335 & 25.2 & 1,866 & 17.8 \\
\hline \multicolumn{5}{|l|}{ Race/Ethnicity } \\
\hline Non-Hispanic white & 67,066 & 83.2 & 9,122 & 87.2 \\
\hline African American & 6,766 & 8.4 & 565 & 5.4 \\
\hline Hispanic white & 2,927 & 3.6 & 390 & 3.7 \\
\hline Asian/Pacific Islander & 2,415 & 3.0 & 195 & 1.9 \\
\hline American Indian/Alaska Native & 345 & 0.4 & 59 & 0.6 \\
\hline Other & 1,133 & 1.4 & 133 & 1.3 \\
\hline \multicolumn{5}{|l|}{ Oral contraceptive use, years } \\
\hline Never & 49,019 & 60.8 & 5,553 & 53.1 \\
\hline$<5$ & 17,445 & 21.6 & 2,963 & 28.3 \\
\hline$\geq 5$ & 14,165 & 17.6 & 1,946 & 18.6 \\
\hline Missing & 23 & & 2 & \\
\hline \multicolumn{5}{|l|}{ Hormone therapy use } \\
\hline Never & 33,464 & 41.5 & 3,346 & 32.0 \\
\hline Former & 11,923 & 14.8 & 1,622 & 15.5 \\
\hline Current unopposed estrogen user & 19,363 & 24.0 & 3,377 & 32.3 \\
\hline Current estrogen and progestin user & 15,827 & 19.6 & 2,102 & 20.1 \\
\hline Missing & 75 & & 17 & \\
\hline \multicolumn{5}{|l|}{ History of a hysterectomy at baseline } \\
\hline No & 47,657 & 59.1 & 5,270 & 50.4 \\
\hline Yes & 32,924 & 40.9 & 5,182 & 49.6 \\
\hline Missing & 71 & & 12 & \\
\hline \multicolumn{5}{|l|}{ Body mass index quartiles, $\mathrm{kg} / \mathrm{m}^{2}$} \\
\hline$<23.21$ & 19,986 & 25.1 & 2,601 & 25.1 \\
\hline 23.21-26.09 & 19,898 & 25.0 & 2,639 & 25.5 \\
\hline $26.10-30.03$ & 19,957 & 25.0 & 2,571 & 24.8 \\
\hline$\geq 30.04$ & 19,866 & 24.9 & 2,547 & 24.6 \\
\hline Missing & 945 & & 106 & \\
\hline \multicolumn{5}{|l|}{ Regular use of any NSAID at baseline, years } \\
\hline No & 53,237 & 66.0 & 6,204 & 59.3 \\
\hline Yes & 27,414 & 34.0 & 4,260 & 40.7 \\
\hline$<3$ & 12,610 & 15.6 & 1,713 & 16.4 \\
\hline$\geq 3$ & 14,804 & 18.4 & 2,547 & 24.3 \\
\hline Missing & 1 & & - & \\
\hline \multicolumn{5}{|l|}{ Current alcohol use } \\
\hline Never & 8,944 & 11.2 & 1,122 & 10.8 \\
\hline Former drinker & 14,723 & 18.4 & 2,292 & 22.0 \\
\hline \multicolumn{5}{|l|}{ Current drinker } \\
\hline$<7$ drinks per week & 45,954 & 57.3 & 6,038 & 58.0 \\
\hline$\geq 7$ drinks per week & 10,497 & 13.1 & 950 & 9.1 \\
\hline Missing & 484 & & 62 & \\
\hline \multicolumn{5}{|l|}{ Smoking status } \\
\hline Never & 40,483 & 50.8 & 5,313 & 51.5 \\
\hline Former & 34,155 & 42.9 & 4,441 & 42.8 \\
\hline Current & 4,987 & 6.3 & 594 & 5.8 \\
\hline Missing & 1,027 & & 140 & \\
\hline \multicolumn{5}{|l|}{$\begin{array}{l}\text { No. of cups of regular coffee consumed } \\
\text { per day }\end{array}$} \\
\hline 0 & 34,514 & 43.6 & 4,578 & 44.6 \\
\hline 1 & 13,862 & 17.5 & 1,727 & 16.8 \\
\hline 2 & 23,177 & 29.3 & 2,958 & 28.8 \\
\hline$\geq 3$ & 7,576 & 9.6 & 999 & 9.7 \\
\hline Missing & 1,523 & & 202 & \\
\hline
\end{tabular}

Abbreviation: NSAID, nonsteroidal anti-inflammatory drug. similar to each other with respect to BMI, smoking status, and consumption of caffeinated coffee.

Women with a migraine history had a lower risk of developing breast cancer (multivariate adjusted HR, $0.89 ; 95 \%$ CI, 0.80 to 0.98 ) than women without a migraine history (Table 2). This association was similar for risks of both in situ and invasive breast cancer and did not vary by histology among invasive occurrences. There was some suggestion that this lower risk was more pronounced among older than younger women, but the interaction with age was not statistically significant $(P$ for interaction $=.08 ; 50$ to 59 years of age at enrollment data: HR, $0.93 ; 95 \%$ CI, 0.78 to $1.11 ; 60$ to 69 years of age at enrollment data: HR, $0.94 ; 95 \%$ CI, 0.81 to 1.10 ; and 70 to 79 years of age at enrollment data: HR, $0.69 ; 95 \% \mathrm{CI}, 0.53$ to 0.90 ). The lower risk was somewhat more pronounced for invasive ER-positive/PR-positive tumors (HR, 0.83; 95\% CI, 0.71 to 0.97 ), as no reduction in risk was observed for invasive ER-negative/PR-negative tumors (HR, 1.16; $95 \% \mathrm{CI}, 0.86$ to 1.57 ), and this difference in risk estimates was borderline statistically significant $(P=.06)$.

Risks of breast cancer overall, invasive breast cancer, and invasive ER-positive/PR-positive breast cancer did not vary appreciably by current use of NSAIDs at baseline, even among current users for 3 years or longer (Table 3 ). Risks also were similar when current use of aspirin and ibuprofen were assessed separately (data not shown). In addition, given that some migraine triggers also are related to breast cancer risk, we assessed the relationship between migraine and risk of ER-positive/PR-positive invasive breast cancer among subgroups of women, including never drinkers of alcohol during the past 10 years, never users of oral contraceptives, and never users of menopausal hormone therapy. We focused on this patient subtype, because this was the subtype most strongly related to migraine history. Within each of these exposure categories, the direction and magnitude of the association between migraine history and ER-positive/PR-positive invasive breast cancer was similar to the overall risk estimate (never drinkers data: HR, $0.79 ; 95 \% \mathrm{CI}, 0.46$ to 1.34 ; never users of oral contraceptives data: $\mathrm{HR}, 0.84 ; 95 \% \mathrm{CI}, 0.69$ to 1.04 ; and never users of menopausal hormone therapy data: HR, 0.86 ; $95 \%$ CI, 0.64 to 1.15 ). The relationship between migraine and breast cancer also did not vary when analyses were stratified by caffeine consumption, another migraine trigger, as measured by daily consumption of caffeinated coffee.

\section{DISCUSSION}

Consistent with two recently published, population-based, casecontrol studies in entirely different populations, ${ }^{1,2}$ we estimate that migraine history is associated with a $10 \%$ lower risk of invasive breast cancer overall and a 17\% lower risk of ER-positive/PR-positive invasive breast cancer. The magnitude of the lower risk we observed is more modest than the $26 \%$ to $33 \%$ lower risk of postmenopausal breast cancer observed previously, ${ }^{1,2}$ and this could be due to greater misclassification of migraine history and nondifferential bias toward the null. Specifically, WHI data were based on self-reported answers to a lengthy self-administered questionnaire, and data were collected in person by trained interviewers in the two prior studies, in which participants were additionally probed regarding age at migraine diagnosis and if they had every used any prescription medications to treat their migraines. As a result, there is likely to be greater misclassification 
Table 2. Risk of Breast Cancer Associated With Migraine History

\begin{tabular}{|c|c|c|c|c|c|c|}
\hline \multirow[b]{2}{*}{ Breast Cancer Type } & \multirow{2}{*}{$\begin{array}{c}\text { No. of Incident Cases } \\
\text { Without a Migraine History }\end{array}$} & \multirow{2}{*}{$\begin{array}{l}\text { No. of Incident Cases With } \\
\text { a Migraine History }\end{array}$} & \multicolumn{2}{|c|}{$\begin{array}{l}\text { Adjusted for Age and } \\
\text { Race/Ethnicity }\end{array}$} & \multicolumn{2}{|c|}{$\begin{array}{l}\text { Multivariate Adjusted } \\
\text { Analysis* }\end{array}$} \\
\hline & & & $\mathrm{HR}$ & $95 \% \mathrm{Cl}$ & $\mathrm{HR}$ & $95 \% \mathrm{Cl}$ \\
\hline Any breast cancer & 3,595 & 411 & 0.89 & 0.80 to $0.98 \dagger$ & 0.89 & 0.80 to $0.98 \dagger$ \\
\hline In situ breast cancer & 623 & 65 & 0.80 & 0.62 to 1.03 & 0.81 & 0.62 to 1.05 \\
\hline Invasive breast cancer & 2,972 & 346 & 0.91 & 0.81 to 1.01 & 0.90 & 0.80 to 1.01 \\
\hline \multicolumn{7}{|l|}{ ER/PR status } \\
\hline ER and PR positive & 1,693 & 185 & 0.85 & 0.73 to $0.99 \dagger$ & 0.83 & 0.71 to $0.97 \dagger$ \\
\hline ER positive and PR negative & 361 & 42 & 0.89 & 0.65 to 1.23 & 0.92 & 0.66 to 1.28 \\
\hline ER and PR negative & 335 & 53 & 1.22 & 0.91 to 1.63 & 1.16 & 0.86 to 1.57 \\
\hline \multicolumn{7}{|l|}{ Histology } \\
\hline Ductal & 1,709 & 207 & 0.94 & 0.81 to 1.08 & 0.91 & 0.79 to 1.06 \\
\hline Lobular & 678 & 76 & 0.87 & 0.69 to 1.10 & 0.88 & 0.69 to 1.13 \\
\hline Other & 359 & 33 & 0.73 & 0.51 to 1.05 & 0.72 & 0.50 to 1.04 \\
\hline
\end{tabular}

Abbreviations: $H R$, hazard ratio; $E R$, estrogen receptor; $P R$, progesterone receptor.

*Multivariate adjusted HRs are adjusted for age, race/ethnicity, and the following baseline characteristics: hysterectomy, use of menopausal hormones, nonsteroidal anti-inflammatory drug use/duration, alcohol consumption, smoking status, and regular coffee consumption. The 3,333 women missing data for one or more of the covariates adjusted for in the multivariate models were excluded from these analyses.

$\dagger P<.05$.

of migraine history in WHI, but this misclassification is nondifferential, which means that women who were or were not diagnosed with breast cancer could be assumed to be equally likely to have migraine history misclassified. It is well established that this type of misclassification results in an attenuation of the estimated size of the true association. ${ }^{17}$ However, a unique strength of WHI was its collection of detailed covariate data, including data on NSAID use, which was lacking in the prior studies. Here, we find that the reduction in risk associated with migraine history did not vary by NSAID use.

Additionally, common triggers for migraines are use of exogenous hormones and alcohol consumption. Given that both of these exposures are positively related to breast cancer risk across numerous studies, ${ }^{1,18,19}$ one potential explanation for the relationship between migraine and breast cancer is simply that migraine sufferers may be less likely to use exogenous hormones and alcohol and that this conveys a lower risk of breast cancer (though in this cohort migraine sufferers were actually more likely to use oral contraceptives and unopposed estrogen hormone therapy). However, we observed the same relationship between migraine and breast cancer when our analyses were restricted to never users of oral contraceptives, never users of menopausal hormone therapy, and never drinkers, similar to the only prior study that also stratified its analyses by these factors. ${ }^{2}$

\begin{tabular}{|c|c|c|c|c|c|c|}
\hline \multirow[b]{2}{*}{ NSAID Use Category } & \multirow{2}{*}{$\begin{array}{c}\text { No. of Incident Cases } \\
\text { Without a Migraine } \\
\text { History }\end{array}$} & \multirow{2}{*}{$\begin{array}{c}\text { No. of Incident Cases } \\
\text { With a Migraine } \\
\text { History }\end{array}$} & \multicolumn{2}{|c|}{$\begin{array}{c}\text { Adjusted for Age and } \\
\text { Race/Ethnicity }\end{array}$} & \multicolumn{2}{|c|}{$\begin{array}{l}\text { Multivariate Adjusted } \\
\text { Analysis* }\end{array}$} \\
\hline & & & $\mathrm{HR}$ & $95 \% \mathrm{Cl}$ & $\mathrm{HR}$ & $95 \% \mathrm{Cl}$ \\
\hline \multicolumn{7}{|l|}{ Not currently using NSAIDs } \\
\hline Any breast cancer & 2,355 & 247 & 0.90 & 0.79 to 1.03 & 0.89 & 0.78 to 1.02 \\
\hline Invasive breast cancer & 1,930 & 211 & 0.94 & 0.82 to 1.09 & 0.93 & 0.80 to 1.07 \\
\hline ER- and PR-positive invasive breast cancer & 1,080 & 105 & 0.83 & 0.68 to 1.02 & 0.79 & 0.64 to $0.98 \dagger$ \\
\hline \multicolumn{7}{|l|}{ Current NSAID users } \\
\hline Any breast cancer & 1,241 & 164 & 0.86 & 0.73 to 1.02 & 0.88 & 0.74 to 1.04 \\
\hline Invasive breast cancer & 1,042 & 135 & 0.85 & 0.71 to 1.02 & 0.87 & 0.72 to 1.04 \\
\hline ER- and PR-positive invasive breast cancer & 613 & 80 & 0.87 & 0.69 to 1.10 & 0.88 & 0.69 to 1.12 \\
\hline \multicolumn{7}{|l|}{ Current NSAID users for $<3$ years } \\
\hline Any breast cancer & 586 & 57 & 0.74 & 0.56 to $0.97 \dagger$ & 0.74 & 0.56 to $0.97 \dagger$ \\
\hline Invasive breast cancer & 488 & 52 & 0.81 & 0.60 to 1.07 & 0.81 & 0.60 to 1.08 \\
\hline ER- and PR-positive invasive breast cancer & 276 & 34 & 0.95 & 0.66 to 1.35 & 0.96 & 0.66 to 1.39 \\
\hline \multicolumn{7}{|l|}{ Current NSAID users for $\geq 3$ years } \\
\hline Any breast cancer & 655 & 107 & 0.96 & 0.78 to 1.18 & 0.98 & 0.79 to 1.21 \\
\hline Invasive breast cancer & 554 & 83 & 0.89 & 0.70 to 1.12 & 0.91 & 0.72 to 1.15 \\
\hline ER- and PR-positive invasive breast cancer & 337 & 46 & 0.81 & 0.60 to 1.11 & 0.83 & 0.61 to 1.14 \\
\hline \multicolumn{7}{|c|}{$\begin{array}{l}\text { Abbreviations: NSAID, nonsteroidal anti-inflammatory drug; HR, hazard ratio; ER, estrogen receptor; PR, progesterone receptor. } \\
\text { "Multivariate adjusted HRs are adjusted for age, race/ethnicity, and the following baseline characteristics: hysterectomy, use of menopausal hormones, alcohol } \\
\text { consumption, smoking status, and regular coffee consumption. The } 3,332 \text { women with a known NSAID use history but with missing data for one or more of the } \\
\text { covariates adjusted for in the multivariate models were excluded from these analyses. } \\
+P<.05 \text {. }\end{array}$} \\
\hline
\end{tabular}


The primary motivation for considering a potential link between migraine and breast cancer is that both diseases are hormonally related. The majority of established risk factors for sporadic breast cancer have a hormonal component; as a result of hormonal changes, migraine attacks can be triggered (eg, with declines in estrogen occurring during the natural menstrual cycle) or suppressed (eg, during the third trimester of pregnancy when estrogen levels reach a high steadystate). ${ }^{6-11}$ Interestingly, in both this study and the two prior studies evaluating the relationship between migraine and breast cancer, a history of migraine appeared to more strongly related to risk of ERpositive/PR-positive breast cancer, supporting a hormonal basis for this relationship. ${ }^{1,2}$ However, the precise biology and hormonal pathways of migraine relevant to a potential reduction in breast cancer risk are poorly understood. Migraine is also a heterogeneous disease; not all are associated with hormonal changes. Thus, additional studies assessing whether the types and triggers of migraine are associated with breast cancer risk could provide greater insight into the biology underlying the relationship between migraine and breast cancer and specifically into the influence of migraine on risk of ER-positive/PRpositive breast tumors.

It is important to acknowledge the limitations of this study. Information on clinical diagnosis of migraine was based solely on participant recall and is subject to potential bias. Underreporting of migraine is of particular concern, because an estimated 27\% to 59\% of migraine sufferers are never clinically diagnosed ${ }^{20-22}$ and, alternatively, because some diagnosed patients may not have suffered headaches that met established clinical criteria for a migraine diagnosis. Although the extent of this type of misclassification is unknown, it is most likely nondifferential, given the prospective nature of this study. Another potential limitation of this study is that migraine history was only ascertained at baseline, so women diagnosed with migraine after baseline were all classified as not having a migraine history. However, a recent report on the cumulative lifetime migraine incidence among women in the United States indicates that $97 \%$ of women ever clinically diagnosed with migraine are diagnosed before age 50 years, ${ }^{23}$ and all women enrolled on the WHI OS were 50 years of age or older, which thus limited the impact of the resulting misclassification. The WHI OS did not collect information on the timing, frequency, or intensity of migraine headaches, so the impact of these factors on breast cancer risk could not be assessed. Lastly, although some data on
NSAID use were available, duration was only known for women who reported currently using NSAIDs at study enrollment. However, on the basis of a recent meta-analysis of studies evaluating the relationship between NSAID use and breast cancer risk, it is unclear the extent to which duration of use is related to the overall $12 \%$ reduced risk of breast cancer risk associated with any NSAID intake. ${ }^{14}$ In an analysis that used WHI OS data, though, only NSAID use for 5 years or longer was associated with a lower risk of breast cancer. ${ }^{15}$

This study provides support for two initial reports from casecontrol studies that migraine is associated with a lower risk of breast cancer. All three studies also consistently observed that this relationship is somewhat stronger for hormone-receptor-positive tumors. ${ }^{1,2}$ Here, we expand our knowledge of this association by demonstrating that this relationship is independent of NSAID use and exposure to common migraine triggers, including exogenous hormones and alcohol. However, additional work is needed to resolve what accounts for this relationship, such as differences in hormonal milieus or sensitivities in migraineurs compared with women who do not suffer from migraines, to convey a lower risk of breast cancer.

\section{AUTHORS' DISCLOSURES OF POTENTIAL CONFLICTS} OF INTEREST

The author(s) indicated no potential conflicts of interest.

\section{AUTHOR CONTRIBUTIONS}

Conception and design: Christopher I. Li, Bette Caan, Rowan T. Chlebowski, Marcia L. Stefanick

Financial support: Rowan T. Chlebowski, Marcia L. Stefanick

Administrative support: Rowan T. Chlebowski, Ross Prentice Provision of study materials or patients: Bette Caan, Mary Jo O'Sullivan, Marcia L. Stefanick, Ross Prentice

Collection and assembly of data: Rowan T. Chlebowski, Ross Prentice Data analysis and interpretation: Christopher I. Li, Elizabeth C. Bluhm, Ross Prentice

Manuscript writing: Christopher I. Li, Robert W. Mathes, Bette Caan, Mary F. Cavanagh, Rowan T. Chlebowski, Yvonne Michael, Mary Jo O’Sullivan, Marcia L. Stefanick, Ross Prentice

Final approval of manuscript: Christopher I. Li, Elizabeth C. Bluhm, Bette Caan, Mary F. Cavanagh, Rowan T. Chlebowski, Yvonne Michael, Mary Jo O’Sullivan, Marcia L. Stefanick, Ross Prentice

\section{REFERENCES}

1. Mathes RW, Malone KE, Daling JR, et al: Migraine in postmenopausal women and the risk of invasive breast cancer. Cancer Epidemiol Biomarkers Prev 17:3116-3122, 2008

2. Li Cl, Mathes RW, Malone KE, et al: Relationship between migraine history and breast cancer risk among premenopausal and postmenopausal women Cancer Epidemiol Biomarkers Prev 18:2030-2034, 2009

3. Silberstein SD: Sex hormones and headache. Rev Neurol (Paris) 156:4S30-4S41, 2000 (suppl 4)

4. Zacur HA: Hormonal changes throughout life in women. Headache 46:S49-S54, 2006 (suppl 2)

5. Martin VT, Lipton RB: Epidemiology and biology of menstrual migraine. Headache 48 :S124S130, 2008 (suppl 3)
6. Sulak PJ, Scow RD, Preece C, et al: Hormone withdrawal symptoms in oral contraceptive users. Obstet Gynecol 95:261-266, 2000

7. Granella F, Sances G, Zanferrari C, et al: Migraine without aura and reproductive life events: A clinical epidemiological study in 1300 women. Headache 33:385-389, 1993

8. Dzoljic $E$, Sipetic $S$, Vlajinac $H$, et al: Prevalence of menstrually related migraine and nonmigraine primary headache in female students of Belgrade University. Headache 42:185-193, 2002

9. Granella F, Sances G, Pucci E, et al: Migraine with aura and reproductive life events: A case control study. Cephalalgia 20:701-707, 2000

10. Kornstein SG, Parker AJ: Menstrual migraines: Etiology, treatment, and relationship to premenstrual syndrome. Curr Opin Obstet Gynecol 9:154159, 1997

11. Sances G, Granella F, Nappi RE, et al: Course of migraine during pregnancy and postpar- tum: A prospective study. Cephalalgia 23:197205, 2003

12. Neri I, Granella F, Nappi $R$, et al: Characteristics of headache at menopause: A clinico-epidemiologic study. Maturitas 17:31-37, 1993

13. Dumitrescu RG, Cotarla I: Understanding breast cancer risk: Where do we stand in 2005? J Cell Mol Med 9:208-221, 2005

14. Takkouche B, Regueira-Mendez C, Etminan $M$ : Breast cancer and use of nonsteroidal antiinflammatory drugs: A meta-analysis. J Natl Cancer Inst 100:1439-1447, 2008

15. Harris RE, Chlebowski RT, Jackson RD, et al: Breast cancer and nonsteroidal anti-inflammatory drugs: Prospective results from the Women's Health Initiative. Cancer Res 63:6096-6101, 2003

16. The Women's Health Initiative Study Group: Design of the Women's Health Initiative clinical trial and observational study. Control Clin Trials 19:61109, 1998 
17. Thomas DC: Re: "When will nondifferential misclassification of an exposure preserve the direction of a trend?" Am J Epidemiol 142:782-784, 1995

18. Collaborative Group on Hormonal Factors in Breast Cancer: Breast cancer and hormone replacement therapy: Collaborative reanalysis of data from 51 epidemiological studies of 52,705 women with breast cancer and 108,411 women without breast cancer. Lancet 350:1047-1059, 1997
19. Hamajima N, Hirose $K$, Tajima $K$, et al: Alcohol, tobacco and breast cancer: Collaborative reanalysis of individual data from 53 epidemiological studies, including 58,515 women with breast cancer and 95,067 women without the disease. Br J Cancer 87:1234-1245, 2002

20. Lipton RB, Stewart WF, Celentano DD, et al: Undiagnosed migraine headaches: A comparison of symptom-based and reported physician diagnosis. Arch Intern Med 152:1273-1278, 1992
21. Lipton RB, Stewart WF: Migraine in the United States: A review of epidemiology and health care use. Neurology 43:S6-S10, 1993

22. Lipton RB, Stewart WF, Simon D: Medical consultation for migraine: Results from the American Migraine Study. Headache 38:87-96, 1998

23. Stewart WF, Wood C, Reed ML, et al: Cumulative lifetime migraine incidence in women and men. Cephalalgia 28:1170-1178, 2008

\section{Not an ASCO Member? Subscribe to Journal of Oncology Practice}

Journal of Oncology Practice (JOP) is ASCO's bimonthly forum for providing its subscribers with information, news, and tools to enhance practice efficiency and promote a high standard of quality for patient care in your practice.

Every issue of $J O P$ includes important features on cancer policy issues and their practical effect on cancer care, methods for enhancing the quality of patient care, and tools for improving practice management.

Whether you are in an office or hospital setting, a community or academic environment, JOP provides practical information and advice that oncologists and other oncology professionals can apply immediately to their practice. Key features include:

- Published for all members of the practice-physicians, nurses, and administrators

- Timely and relevant information to help practices succeed

- Focus on improving practice efficiency and quality of care

- Covers legal, financial, technology, and personnel issues

Subscribe today at www.jop.ascopubs.org

ASC()

American Society of Clinical Oncology 